PROCEEDINGS OF THE

AMERICAN MATHEMATICAL SOCIETY

Volume 135, Number 11, November 2007, Pages 3499-3505

S 0002-9939(07)08966-6

Article electronically published on July 27, 2007

\title{
DIMENSION DES FAMILLES DE COURBES LISSES SUR UNE SURFACE QUARTIQUE NORMALE DE $\mathbb{P} 3$
}

\author{
SÉBASTIEN GUFFROY \\ (Communicated by Michael Stillman)
}

\begin{abstract}
RÉSUMÉ. Dans cette note, on montre que les courbes, lisses connexes, de degré $d$ et genre $g$, tracées sur une surface quartique normale variable de $\mathbb{P}_{3}$, et n'y étant pas intersection complète, forment des familles de dimensions $\leqslant g+33$. Cette majoration est la meilleure possible. Comme application on prouve que le schéma de Hilbert des courbes lisses connexes de $\mathbb{P}_{3}$ de degré 12 et genre 13 est irréductible.
\end{abstract}

\section{INTRODUCTION}

On travaille sur le corps des complexes et l'on note $\mathcal{H}_{d, g}$ le schéma de Hilbert des courbes lisses connexes de $\mathbb{P}_{3}$ de degré $d$ et genre $g$. Soit $n(d, g, s)=\max _{V_{s}}\left\{\operatorname{dim} V_{s}\right\}$ où le maximum est pris sur les sous-ensembles (maximaux) $V_{s} \subset \mathcal{H}_{d, g}$ dont les points représentent des courbes tracées sur une surface normale de degré $s$.

Il est évident que $n(d, g, 1)=\left(\begin{array}{c}d+2 \\ 2\end{array}\right)+2(d>1)$ et que $n(d, g, 2)=2 d+g+8$ $(d>4)$. Les courbes tracées sur un cône cubique normal se répartissent en deux familles ([5, Prop 2.12]) dont on vérifie facilement qu'elles sont de dimensions $\leqslant$ $d+g+12$. Celles sur les cubiques normales non réglées formant des familles de dimensions $\leqslant d+g+18$ d'après [6. Prop 3.1], on trouve que $n(d, g, 3)=d+g+18$ $(d>9)$.

Dans [2], Ellia montre que les courbes lisses connexes de degré $d$ et genre $g$ tracées sur une quartique à points doubles appartiennent à des familles de dimensions $\leqslant g+39$. D'autre part, le théorème de Noether-Lefschetz entraine que toute courbe sur une quartique générale y est intersection complète. Dans ce cas, on obtient une famille de dimension $g+34(d>16)$.

Dans cette note, on achève l'étude de $n(d, g, 4)$ en prouvant que, les courbes lisses connexes de degré $d$ et genre $g$ contenues dans une quartique normale variable, sans y être intersection complète, constituent des familles de dimensions $\leqslant g+33$. C'est le résultat à la fois attendu et optimal : les familles de courbes sur une surface lisse fournissant l'égalité.

Les majorations de $n(d, g, s)$ sont utiles, par exemple, pour identifier une composante irréductible du schéma de Hilbert. On montre au second numéro comment la borne $g+33$ permet de vérifier que $\mathcal{H}_{12,13}$ est irréductible conformément à une conjecture de Gruson.

Received by the editors June 1, 2005 and, in revised form, August 23, 2006.

2000 Mathematics Subject Classification. Primary 14J17, 14 N15.

(C)2007 American Mathematical Society 


\section{LA MAJORATION $g+33$}

Dans cette section on justifie la

Proposition 1.1. Les courbes lisses connexes de degré d et genre g, tracées sur une quartique normale variable de $\mathbb{P}_{3}$, et qui n'y sont pas intersection complète, forment des familles de dimensions $\leqslant g+33$.

La démonstration n'est en substance que la réécriture de celle d'Ellia dans [2] à la lumière d'un théorème de Lejeune-Jalabert et Gonzales-Springer non disponible à l'époque et énoncé après.

Pour majorer les dimensions de ces familles de courbes on procède comme dans [2, III]. Tout d'abord, on distingue les surfaces quartiques normales en fonction de leur(s) singularité(s) non rationnelle(s) en suivant la classification d'Umezu [13. Pour une quartique normale $S \subset \mathbb{P}_{3}$, on note $\pi: \widetilde{S} \rightarrow S$ sa désingularisation minimale, $I$ l'ensemble de ses points singuliers non rationnels, et $q$ l'irrégularité de $\widetilde{S}$. Alors on est dans l'un des cas suivants :

1. $I_{0}=\varnothing, S$ est lisse ou bien $\widetilde{S}$ est une surface $K 3$.

2. $I_{1}=\{x\}, p_{g}(x)=4, q=3$. $S$ est alors un cône de sommet $x$.

3. $I_{2}=\{x\}, p_{g}(x)=1, q=0$. La singularité $x$ est minimale elliptique [7].

4. $I_{3}=\{x, y\}, p_{g}(x)=p_{g}(y)=1, q=1$. Avec $x$ et $y$ simples elliptiques de même type.

5. $I_{4}=\{x\}, p_{g}(x)=2, q=1$. Il y a 4 types possibles [13, Prop 2].

Pour chaque ensemble $I_{j}$, les quartiques avec $I=I_{j}$ définissent un sous-ensemble de $\mathbb{P}_{34}$, l'espace de paramètres des quartiques de $\mathbb{P}_{3}$, dont la dimension est notée $D_{j}$.

Ensuite 11 une telle surface $S$ étant fixée, on se munit d'une courbe lisse connexe $C \subset S$, de degré $d$ et genre $g$, dont on note $\widetilde{C}$ la transformée propre par $\pi$. Le fibré $\mathrm{E}=\mathscr{O}_{\widetilde{S}}(\widetilde{C})$ s'inscrit dans la suite exacte $0 \rightarrow \mathrm{E}^{\vee} \rightarrow \mathscr{O}_{\widetilde{S}} \rightarrow \mathscr{O}_{\widetilde{C}} \rightarrow 0$ qui, une fois tensorisée par $\omega_{\widetilde{S}}$, induit en cohomologie $H^{1} \omega_{\widetilde{S}} \mid \widetilde{C} \rightarrow H^{2}\left(\mathrm{E}^{\vee} \otimes \omega_{\widetilde{S}}\right) \rightarrow H^{2} \omega_{\widetilde{S}} \rightarrow 0$. On a $\omega_{\widetilde{S}} \mid \widetilde{C} \simeq \mathscr{O}_{\widetilde{C}}(-\mathcal{D})$ pour un diviseur $\mathcal{D} \geqslant 0$ de degré $Z_{k} \cdot \widetilde{C}$ où $Z_{k}$ est le cycle anticanonique défini par $\omega_{\widetilde{S}}=\mathscr{O}_{\widetilde{S}}\left(-Z_{k}\right)$. La dualité de Serre sur $\widetilde{C}$ donne $h^{1} \omega_{\widetilde{S}} \mid \widetilde{C}=h^{0} \omega_{\widetilde{C}}(\mathcal{D})$. Quand $\mathcal{D}>0, \omega_{\widetilde{C}}(\mathcal{D})$ est non spécial et le nombre de ses sections globales indépendantes est donné par Riemann-Roch. Sinon $\mathcal{D}=0$, et l'on a $h^{0} \omega_{\widetilde{C}}(\mathcal{D})=h^{0} \omega_{\widetilde{C}}=g$. Autrement dit :

$$
h^{0} \mathrm{\textrm {L }} \leqslant g+ \begin{cases}Z_{k} \cdot \widetilde{C} & \text { si } C \text { passe par une singularité irrationnelle de } S, \\ 1 & \text { sinon. }\end{cases}
$$

D'après [2, preuve Cor. III.2], les courbes sur ce type de surface forment une famille de dimension inférieure à

(1) $q+D_{j}+ \begin{cases}g+Z_{k} \cdot \widetilde{C}-1 & \text { si } C \text { passe par une singularité irrationnelle de } S, \\ g & \text { sinon. }\end{cases}$

On ajoute $q$ pour tenir compte des systèmes continus de courbes non linéairement équivalentes.

\footnotetext{
${ }^{1}$ Le calcul suivant, pris verbatim de [2], est inclus pour la commodité du lecteur.
} 
Reste à controler la quantité $Z_{k} \cdot \widetilde{C}$. La courbe lisse $\widetilde{C}$ intercepte au plus une composante irréductible de chaque composante connexe de $Z_{k}$ et ce transversalement. Il s'agit donc de majorer les multiplicités de ces composantes irréductibles dans $Z_{k}$. Le problème est local : il ne dépend que du type du point singulier et de la façon dont $C$ y passe. Les composantes irréductibles de $Z_{k}$ à considérer sont caractérisées dans le

Théorème 1.2 (4, Lejeune-Jalabert et Gonzales-Springer, Th 5-2]). Soit (S,p) un germe de surface normale singulier, et $C \subset S$ un germe de courbe lisse passant par $p$. Les notations $\pi, \widetilde{S}$, et $\widetilde{C}$ sont comme précédemment. Soit $\mathfrak{m}$ l'idéal maximal de $\mathscr{O}_{S, p}$ et $Z_{f}$ la composante de codimension 1 du cycle sous-jacent au sous-schéma de $\widetilde{S}$ défini par $\mathfrak{m} \mathscr{O}_{\widetilde{S}}$.

Si $Z_{f}=\sum n_{i} \mathscr{A}_{i}$ où les $\mathscr{A}_{i}$ sont intègres et distincts, l'unique indice $i_{0}$ tel que $\widetilde{C} \cdot \mathscr{A}_{i_{0}}>0$ est tel que $n_{i_{0}}=1$. Réciproquement, pour tout $i$ tel que $n_{i}=1$, il existe une courbe lisse dont le transformé strict coupe transversalement $\mathscr{A}_{i}$.

On évalue maintenant (1) en fonction des types $I_{j}$ :

1. $I=\varnothing: S$ est lisse ou $\widetilde{S}$ est une surface $K 3$. Alors $q=0$ et $h^{0} \mathcal{L}=g+1$. Les courbes étudiées n'étant pas intersection complète par hypothèse, on a $D_{1} \leqslant 33$ par le théorème de Noether-Lefschetz d'où la borne attendue. On a l'égalité pour les courbes tracées sur une unique quartique lisse 2

2. $S$ est un cône sur une quartique plane lisse $\gamma$. La seule singularité de $S$ est son sommet. Ces surfaces forment une famille de dimension 17. $\widetilde{S}$ s'identifie à la surface réglée $\mathbb{P}\left(\mathscr{O}_{\gamma} \oplus \mathscr{O}_{\gamma}(-4)\right)$. Si $C_{0}$ est l'image réciproque du sommet $x$ de $S$, on a pour l'équivalence numérique $Z_{k} \equiv 2 C_{0}$, et $\widetilde{C} \cdot Z_{k}=2$ ou 0 suivant que $C$ passe par $x$ ou non. On obtient une majoration des dimensions des familles de courbes sur ce type de surface par $g+21$.

3. $I=\{x\}$ où $x$ est minimal elliptique. D'après [7, Th 3.13] $Z_{k}=Z_{f}$, il suit alors du théorème 1.2 que $Z_{k} \cdot \widetilde{C} \leqslant 1$. Comme $q=0$, il vient que les familles de courbes sur ce type de surface sont de dimensions $\leqslant g+33$.

4. $S$ possède deux points simples elliptiques. Alors $q=1$ et, comme $Z_{k}=Z_{f}=$ $E_{1} \cup E_{2}$ pour deux courbes elliptiques $E_{1}$ et $E_{2}$, il suit $Z_{k} \cdot \widetilde{C} \leqslant 2$. Les courbes sur ces surfaces forment des familles de dimensions $\leqslant g+2+D_{4}$.

5. $p_{g}(x)=2, q=1$. Il y a quatre sous-cas [13, Prop 2]. Dans chacun d'eux les multiplicités des composantes irréductibles de $Z_{k}$ sont $\leqslant 2$ donc $Z_{k} \cdot \widetilde{C} \leqslant 2$. Les courbes sur ces surfaces constituent ainsi des familles de dimensions $\leqslant g+2+D_{5}$.

Il reste à s'assurer que $\max \left\{D_{4}, D_{5}\right\} \leqslant 31$. Les surfaces $S$ dont les singularités sont comme en 4 ou 5 sont décrites par les théorèmes 1 et 2 de [13]. Pour construire $S$ comme dans le théorème 1 de 13$]$ il faut choisir :

- une courbe elliptique lisse $E$,

- un fibré en droites $L$ de degré 2 sur $E$,

- prendre $P_{1}, P_{2}, P_{3}$ sur $\widetilde{S}=\mathbb{P}\left(\mathscr{O}_{E} \oplus L\right)$ (en fait assujettis à certaines conditions).

Le morphisme $\widetilde{S} \rightarrow \mathbb{P}_{3}$ d'image $S$ est déterminé par ces données. On peut majorer (grossièrement) $D_{4}$ par 1 (choix de $\left.E\right)+1$ (celui de $\left.L\right)+6\left(\right.$ choix des $\left.P_{i}\right)+15$ pour

\footnotetext{
${ }^{2}$ Les courbes tracées sur les quartiques générales forment une famille de dimension $g+34$ par ces mêmes calculs. On retrouve ainsi le résultat mentionné dans l'introduction pour les intersections complètes.
} 
un changement de coordonnées dans le $\mathbb{P}_{3}$ d'arrivée. Pour les surfaces décrites dans le théorème 2 de [13], on a de la même manière $D_{5} \leqslant 19$. D'où une majoration par $g+25$ dans les cas 4 et 5 ce qui achève de prouver la proposition.

\section{Une application : L'irréductibilité de $\mathcal{H}_{12,13}$}

Théorème 2.1. Le schéma $\mathcal{H}_{12,13}$ est irréductible.

Preuve. Soit $C$ une courbe lisse connexe de degré 12 et genre 13 et $[C]$ le point la représentant dans $\mathcal{H}_{12,13}$. On stratifie le schéma de Hilbert suivant les cohomologies possibles pour $C$. Comme toute composante irréductible de $\mathcal{H}_{12,13}$ est de dimension $\geqslant 48$, une strate de dimension $<48$ ne peut contenir de courbe générale. Par cet argument, on vérifie que seule la strate des courbes à cohomologie semi-naturelle est de dimension suffisante pour abriter une courbe générale. On s'assure ensuite de son irréductibilité.

On remarque que $\mathscr{O}_{C}(n)$ est non spécial pour $n \geqslant 3$ et l'on note la fonction de Rao d'une courbe $X$ par $\rho_{X}$ i.e. $\rho_{X}(n)=h^{1} \mathscr{I}_{X}(n)$. Par liaison $s \times t$ on entend une liaison par l'intersection complète de deux surfaces, sans composante commune, de degrés respectifs $s$ et $t$. Désormais $C$ est supposée générale.

(a) $C$ est linéairement normale et n'est pas tracée sur une cubique.

On commence par montrer que $C$ n'est pas située sur une surface cubique. En premier lieu il n'y pas de courbes en ces degré et genre sur une surface de degré $\leqslant 2$. On examine ensuite les courbes contenues dans une cubique normale $S$. Il ne peut s'agir d'un cône puisque chaque courbe lisse sur ces surfaces est arithmétiquement Cohen-Macaulay d'après [5, Prop 2.12]. Il s'ensuit que les familles des courbes tracées sur une cubique normale ont des dimensions $\leqslant d+g+18=43<48$ ([6, Prop 3.1]). D'autre part, celles situées sur une cubique réglée à droite double, elles en interceptent alors $k=3$ fois la génératrice générale, constituent une famille de dimension $2 d+g+12-k=46<48$ d'après [5, Prop B.2]. Enfin, il n'y a pas de courbes de degré 12 et genre 13 sur d'autres types de surfaces cubiques intègres à l'exception des cônes à droite double mais la majoration précédente vaut encore pour ces surfaces.

Si $C$ n'est pas linéairement normale, elle est projection d'une courbe de $\mathbb{P}_{4}$ qui est située sur deux hyperquadriques dont l'intersection se projette en une quartique puisque $C$ n'est pas tracée sur une cubique. Cette quartique est une del Pezzo, éventuellement dégénérée, et l'on sait, voir [1. Prop 2.6], que la dimension de la famille des courbes de degré $d$ et genre $g$ sur ce type de surface est majorée par $d+g+20=45<48$.

(b) $\mathscr{O}_{C}(2)$ n'est pas spécial.

Sinon on est dans l'un des cas d'égalité du théorème de Clifford. On prouve tout d'abord que les courbes hyperelliptiques ne sont pas générales dans $\mathcal{H}_{12,13}$. Dans $\mathcal{M}_{13}$, l'espace de modules des classes d'isomorphismes des courbes lisses de genre 13, on voit le lieu hyperelliptique comme le quotient du schéma de Hurwitz, celui paramètrant les revêtements doubles de $\mathbb{P}_{1}$ à diviseur de ramification de degré $2 g+2$, par le groupe $P G L_{2}(\mathbb{C})$. Il s'ensuit qu'il est de dimension $2 g-1=25$. Une fois fixée une classe $[C] \in \mathcal{M}_{13}$ avec $C$ hyperelliptique, le morphisme $C \rightarrow \mathbb{P}_{3}$ est déterminé par un $g_{12}^{3}$ sur $C$. Le choix de ce $g_{12}^{3}$ repose sur 6 degrés de liberté. En effet, $C$ étant hyperelliptique, chaque $g_{12}^{3}$ est de la forme $3 g_{2}^{1}+p_{1}+\cdots+p_{12-2 \times 3}$. Il convient d'ajouter 15 pour un changement de coordonnées dans le $\mathbb{P}_{3}$ d'arrivée, 
ainsi la dimension de la famille des courbes hyperelliptiques dans $\mathcal{H}_{12,13}$ est-elle $46<48$.

Si $C$ n'est pas hyperelliptique avec $\mathscr{O}_{C}(2)$ spécial alors $\omega_{C} \simeq \mathscr{O}_{C}(2)$. Il s'ensuit que $C$ est le schéma des zéros d'une section d'un fibré $E$ de rang 2 sur $\mathbb{P}_{3}$ :

$$
0 \rightarrow \mathscr{O}_{\mathbb{P}_{3}}\left(-c_{1}\right) \rightarrow E\left(-c_{1}\right) \rightarrow \mathscr{I}_{C} \rightarrow 0 .
$$

De plus il vient $c_{2}=12$ et $c_{1}=6$. Comme $c_{1}>0$ et $h^{0} \mathscr{I}_{C}(3)=0, E$ est stable. Le fibré normalisé associé, que l'on note $F$, a pour classes de Chern $c_{1}=0$ et $c_{2}=3$. Puisque $\rho_{C}(1)=0$, on a $h^{1} F(-2)=0$ et $F$ est un instanton. D'après Ellingsrud et Strømme [3], l'espace de modules de ces fibrés est de dimension attendue 21 . D'autre part, $\operatorname{Ext}^{1}\left(\mathscr{I}_{C}, \mathscr{O}_{\mathbb{P}_{3}}(-6)\right) \simeq H^{2} \mathscr{I}_{C}(2) \simeq H^{1} \omega_{C}$ est de dimension 1. Reste à calculer $h^{0} E=h^{0} \mathscr{I}_{C}(6)+1$. On distingue deux cas :

(i) $h^{0} \mathscr{I}_{C}(4)>0: \quad C$, par le biais d'une bilaison élémentaire de type $(4,-2)$, donne naissance à une une courbe localement de Cohen-Macaulay (LCM) $\Gamma$ de degré 4 et genre arithmétique -3 . Il vient alors que $\rho_{\Gamma}(-1)=\rho_{C}(1)=0$ donc $\Gamma$ n'est pas extrémale et les bornes de Nollet $\left(\left[10\right.\right.$, Th 2.11]) fournissent $\rho_{\Gamma}(4) \leqslant 1$ i.e. $\rho_{C}(6) \leqslant 1$. Ainsi $h^{0} \mathscr{I}_{C}(6) \leqslant 25$.

(ii) $h^{0} \mathscr{I}_{C}(4)=0: C$ est liée, par une liaison $5 \times 5$, à une courbe LCM, $\Gamma$, de degré 13 et genre arithmétique 16 . La suite de définition de $\Gamma$ donne $h^{0} \mathscr{I}_{\Gamma}(3)+$ $h^{1} \mathscr{O}_{\Gamma}(3)=\rho_{\Gamma}(3)-4=\rho_{C}(3)-4$. Mais, $C$ n'est pas tracée sur une surface cubique et $\mathscr{O}_{C}(3)$ n'est pas spécial donc $\rho_{C}(3)=4$, d'où il suit que $h^{0} \mathscr{I}_{\Gamma}(3)=0$. On montre maintenant que $\rho_{\Gamma}(1)=0$ en utilisant le résultat suivant de Schlesinger :

Lemme 2.2 ([12, Cor 3.4.5.]). Soit $\Gamma \subset \mathbb{P}_{3}$ une courbe LCM. On note $R_{1}$ le $\mathbb{C}$-espace vectoriel engendré par les coordonnées de $\mathbb{P}_{3}$ et $t$ un entier. Soit $m \in$ $H^{1} \mathscr{I}_{\Gamma}(t)-\{0\}$ et $\beta: R_{1} \rightarrow H^{1} \mathscr{I}_{\Gamma}(t+1)$ l'application linéaire définie par $h \mapsto h \cdot m$. Si $\operatorname{dim} \operatorname{Ker}(\beta) \geqslant 3$ alors $h^{0} \mathscr{I}_{\Gamma}(t+2)>0$.

Comme $\rho_{\Gamma}(2)=1\left(=\rho_{C}(4)\right)$, le lemme ci-dessus appliqué à $t=1$ donne soit $h^{0} \mathscr{I}_{\Gamma}(3)>0$ soit $\rho_{\Gamma}(1)=0$. On a vu que le premier cas ne se présente pas d'où $\rho_{\Gamma}(1)=\rho_{C}(5)=0$. Par régularité, il vient que $\rho_{C}(6)=0$ d'où $h^{0} \mathscr{I}_{C}(6)=24$.

En conclusion, les courbes avec $\mathscr{O}_{C}(2)$ spécial se répartissent en des familles de dimensions $\leqslant 46$ et (b) est justifié.

(c) C n'est pas tracée sur une surface quartique.

À ce stade on peut supposer que $\rho_{C}(n)=0$ pour $n \leqslant 1, \rho_{C}(2)=2$, et $\rho_{C}(3)=4$. On procède par l'absurde en ajoutant l'hypothèse $h^{0} \mathscr{I}_{C}(4) \geqslant 1$ i.e. $\rho_{C}(4) \geqslant 2$. On précise un peu la cohomologie de $C$ aux points (i) et (ii) puis on en déduit en (iii) une contradiction avec le fait que $C$ soit générale.

(i) $C$ est tracée sur une unique quartique : $\operatorname{Si} h^{0} \mathscr{I}_{C}(4) \geqslant 2$, une liaison $4 \times 4$ donne lieu à une courbe $\Gamma$ de degré 4 et de genre $p_{a}(\Gamma)=-3$. Comme $h^{0} \mathscr{I}_{\Gamma}(2)=$ $h^{1} \mathscr{O}_{C}(2)=0, \Gamma$ n'est pas extrémale et $[\Gamma]$ appartient à une composante du schéma de Hilbert de dimension $\leqslant 19$ par [11, Th 6.2]. Ensuite, $\rho_{\Gamma}(4)=\rho_{C}(0)=0$ et $\mathscr{O}_{\Gamma}(4)$ non spécial entrainent $h^{0} \mathscr{I}_{\Gamma}(4)=15$. La famille des courbes en cours d'étude est de dimension $\leqslant 19$ (celle des courbes $\Gamma$ auxquelles elles sont liées) $+2\left(h^{0} \mathscr{I}_{\Gamma}(4)-h^{0} \mathscr{I}_{C}(4)\right)$ d'après [8, Cor 3.8 p149] soit un total $\leqslant 45$.

(ii) $C$ est 7-régulière.

Lemme 2.3. Soit $C \subset \mathbb{P}_{3}$ une courbe lisse connexe, $H$ un plan général, $Z=H \cap C$, et $\mathscr{I}_{Z}$ son faisceau d'idéaux dans $\mathscr{O}_{H}$. La fonction $\rho_{C}$ est strictement décroissante à partir de $n_{0}+1$ avant de s'annuler où $n_{0}=\max \left\{n \mid h^{1} \mathscr{I}_{Z}(n-1) \neq 0\right\}$. 
Preuve. Le faisceau $\mathscr{I}_{Z}\left(n_{0}+1\right)$ est engendré par ses sections puisqu'il est $\left(n_{0}+1\right)$ régulier au sens de Castelnuovo-Mumford par hypothèse. Il vient en particulier de la suite exacte $0 \rightarrow \mathscr{I}_{C}(n-1) \rightarrow \mathscr{I}_{C}(n) \rightarrow \mathscr{I}_{Z}(n) \rightarrow 0$ que la multiplication par l'équation de $H, \alpha_{n}: H^{1} \mathscr{I}_{C}(n-1) \rightarrow H^{1} \mathscr{I}_{C}(n)$, est surjective pour tout $n \geqslant n_{0}$. Si $\operatorname{Ker}\left(\alpha_{n}\right)=0$ pour un $n \geqslant n_{0}+1$ alors $\operatorname{Ker}\left(\alpha_{m}\right)=0$ pour tout $m \geqslant n$ car c'est un quotient de $H^{0} \mathscr{I}_{Z}(n)$ et $\mathscr{I}_{Z}(n)$ est engendré par ses sections. Comme $\rho_{C}$ est à support fini, on obtient que $\rho_{C}$ est strictement décroissante avant de s'annuler.

La postulation de $C$ force son caractère numérique à être $(5,5,4,4)$. Il s'ensuit que $n_{0}=4$ et le lemme ci-dessus entraine que $\rho_{C}(5) \leqslant 1$ puis $\rho_{C}(6)=0$ d'où la 7-régularité de $C$.

(iii) La proposition 1.1 nous indique que la quartique contenant $C$ n'est pas normale. En s'appuyant sur la classification des quartiques non normales d'Urabe ([14]), $C$ peut être tracée sur une surface réglée sur une courbe elliptique, sur une quartique rationnelle à droite double, ou bien sur un cône sur une quartique plane de genre géométrique 1 . On examine ces possibilités successivement.

Si $C$ est tracée sur une quartique $S$ réglée sur une courbe elliptique $G, S$ n'a pas de point singulier isolé ou immergé. Soit $\mathcal{Q}$ la famille des quartiques de ce type. La normalisation de $S$ est alors sa désingularisation minimale et les calculs de 2, IV 4 Lemme] s'adaptent à cette situation : les courbes contenues dans ce genre de surface quartique forment une famille de dimension $\leqslant \operatorname{dim} \mathcal{Q}+g+r+q(\widetilde{S})$ où $r$ est la longueur du schéma 0 -dimensionnel $C \cap \operatorname{Sing} S$. Il y a deux constructions possibles de ces surfaces en fonction de leur modèle lisse $Z$, voir [14, II 1-2]. Cependant, pour l'équivalence numérique, et en notant $L$ la classe du fibré tautologique sur $Z$ et $f$ celle d'une fibre, il vient que $C$ est numériquement équivalente à $3 L$ ou à $4 L-4 f$. Dans les deux cas le lieu non normal est de classe $-K_{\widetilde{S}}$ et $C \cdot\left(-K_{\widetilde{S}}\right)=12$. Les descriptions des surfaces de la famille $\mathcal{Q}$ de [14, II 1-2] permettent de vérifier que $\operatorname{dim} \mathcal{Q} \leqslant 1+2+2+15=20$. Enfin, pour $[S] \in \mathcal{Q}$, on a $q(\widetilde{S})=1$, et l'on aboutit à une dimension $\leqslant 21+13+12=46<48$ pour la famille des courbes en cours d'étude. Ces dernières ne sont donc pas générales.

Le cas des courbes sur un cône ne pose pas plus de difficultés et se traite de la même manière. On considère désormais les courbes situées sur une quartique rationnelle à droite double. Afin que $C$ soit générale, elle doit être 7-régulière et n'admet donc pas de 8-sécante. Le lemme [2, IV-4 Lemme] nous donne que ces courbes forment une famille de dimension $\leqslant g+7+24=44$. On a vérifié qu'aucune quartique ne contient de courbe générale de degré 12 et genre 13 .

(d) Les courbes à cohomologies semi-naturelle forment une famille irréductible.

On ajoute la condition $h^{0} \mathscr{I}_{C}(4)=0$ à celles de $(c)$. Une liaison $5 \times 5$ donne, par le même argument qu'en (b) (ii), que $\rho_{C}(5)=0$. Il suit que $C$ a la cohomologie semi-naturelle. Pour prouver que ces courbes sont représentées par un sous-schéma irréductible du schéma de Hilbert il suffit d'appliquer le résultat suivant de MartinDeschamps et Perrin, voir 9] : Soient $q$ et $r$ deux entiers positifs tels que $1 \leqslant r$ et $4 q \geqslant 6 r+1$, et $D$ une courbe LCM dont les valeurs non nulles de la fonction de Rao sont $\rho_{D}(n)=r, \rho_{D}(n+1)=q$ et $\rho_{D}(n+2)=1$ pour un certain entier $n$. Alors la famille $\mathcal{F}$ des courbes LCM de même cohomologie que $D$ est irréductible. Par suite l'ouvert de $\mathcal{F}$ paramétrant les courbes lisses connexes est lui aussi irréductible. 
Remarque 2.4. La conjecture évoquée dans l'introduction est la suivante : Le schéma de Hilbert des courbes lisses connexes de degré $d$ et genre $g$ de $\mathbb{P}_{3}$ est irréductible si $g \leqslant 2 d-9$.

\section{REMERCIEMENTS}

Ce travail a été effectué lors de mon séjour au département de Mathématiques de l'Université de Gênes grâce au support du réseau RTN HPRN-CT-2002-00287. Sa rédaction a notablement bénéficié des remarques du rapporteur.

\section{BIBLIOGRAPHIE}

[1] A. Dolcetti et G. Pareschi : On linearly normal space curves, Math. Z., 198 (1988) 73-82 MR938030 (89d:14037)

[2] P. Ellia : D'autres composantes non réduites de Hilb( $\left.\mathbb{P}^{3}\right)$, Math. Ann., 277 (1987) 433-446 MR.891584(88m:14002)

[3] G. Ellingsrud et S. A. Strømme : Stable rank-2 vector bundles on $\mathbb{P}_{3}$ with $c_{1}=0$ and $c_{2}=3$, Math. Ann., 255 (1981), no. 1, 123-135. MR611278 (82e:14023)

[4] G. Gonzalez-Springer et M. Lejeune-Jalabert : Progress in Math., Vol 134 (1996) 9-31, Birkhaüser.

[5] L. Gruson et C. Peskine. Genre des courbes de l'espace projectif(II). Ann. scient. Ec. Norm. Sup., 15 (1983) 401-418. MR690647 (84j:14034)

[6] S. Guffroy. Sur l'incomplétude de la série linéaire caractéristique d'une famille de courbes planes à nœuds et à cusps. Nagoya Math. J., 171 (2003). MR2002013 (2005h:14006)

[7] H. Laufer : On minimally elliptic singularities, Am. J. Math., 99(6) (1977) 1257-1295 MR $0568898(58: 27961)$

[8] M. Martin-Deschamps et D. Perrin. Sur la classification des courbes gauches. Astérisque 184-185. Publ. S.M.F, 1990. MR.1073438 (91h:14039)

[9] M. Martin-Deschamps et D. Perrin : Courbes gauches et module de Rao, J. reine angew. Math., 439 (1993) 103-145 MR.1219697 (94e:14040)

[10] S. Nollet. Subextremal Curves. Manuscripta Math., 94 (1997) 303-317. MR:1485438 (98m:14033)

[11] S. Nollet. et E. Schlesinger : the Hilbert scheme of degree four curves, Compo. Math., 139 (2004) 169-196 MR2025805 (2005b:14010)

[12] E. Schlesinger : The spectrum of projective curves, Ph.D. thesis, University of California at Berkeley (1996)

[13] Y. Umezu : Quartic surfaces of elliptic ruled type, Trans. Am. Math. Soc., 283(1) (1984) 127-143 MR735411 (85c:14024)

[14] T. Urabe : Classification of non-normal quartic surfaces, Tokyo J. Math., 9(2) (1986) 265-295 MR875189 (88a:14041)

Dipartimento di Matematica, Università degli Studi di Genova, Via Dodecaneso, 35. 16146 Genova, Italia

E-mail address: guffroy@math.univ-lille1.fr 